NEWS AND COMMENTARY

TNF Polymorphism and Cardiovascular Disease

\title{
TNF gene polymorphism and quantitative traits related to cardiovascular disease: getting to the heart of the matter
}

George D Mellick

European Journal of Human Genetics (2007) 15, 609-611.

doi:10.1038/sj.ejhg.5201816; published online 21 March 2007

$\mathrm{E}$ xhaustive efforts to relate common genetic variation to complex clinical outcomes such as cardiovascular disease have yielded little in terms of reproducible factors with even modest influences on actual disease risk. Many pundits question the value of association studies to reveal the important genetic determinants for complex diseases, despite the advances in genotyping technology, bioinformatics resources and statistical approaches that now enable whole-genome association analyses to be performed. ${ }^{1-3}$ None-the-less, it is becoming clear that genetic variability accounts for much of the gene-expression variation in human populations ${ }^{4,5}$ and no doubt, a significant proportion of the biological variance and cumulative risk for many important health outcomes. Thus, advancing our basic understanding into how the human genome shapes biological function at a cellular, tissue, system and whole-body level is crucial to ultimately unravelling the determinants of complex diseases seen in human populations.

In the current issue of this journal, Hong et $a l^{6}$ report the results of an alternative examination of data collected from a typical large-scale association study in an attempt to better understand how common genetic variables around the $T N F$ locus relate to clinical and biometric measures commonly examined in studies of cardiovascular disease.

The study genotyped five SNPs in the promoter region of the TNF gene in 1209 subjects with a history of a single myocardial infarction (MI) and 1561 population-based controls without a history of MI. The researchers determined that two of the markers, rs1800630 and rs1800629 in the dbSNP database (http://www. ncbi.nl.nih.gov/SNP/), delineated the most common haplotypes in the Swedish population, estimated diplotype configurations of these markers for individual subjects and proceeded to assess relationships between this genetic variable and 18 quantitative phenotypes traditionally examined in relation to cardiovascular disease. ${ }^{6}$

Interestingly, the analysis provided evidence for association between TNF haplotype and plasma levels of plasminogen activator factor inhibitor 1 (PAI-1). Two other phenotypes, the plasma level of C-reactive protein and the homoeostasis model assessment (HOMO), also showed suggestive relationships; these were not statistically significant upon Bonferroni's correction for multiple testing. The TNF diplotype accounted for $10 \%$ of the variance in PAI-1 plasma levels, surprisingly exceeding the influence of established functional genetic variables in the promoter of SERPINE1 (the gene that codes for the PAI-1 protein, $2 \%$ of the variance). Intriguingly, the haplotype-phenotype relationship was prominent in the group of subjects with a history of MI but not statistically significant in the control group. ${ }^{6}$

The gene coding for TNF-alpha (tumour necrosis factor, $T N F$ ) is located within the highly polymorphic MHC region of chromosome 6, which encodes various genes involved in immunological responses. TNF-alpha is a crucial proinflammatory cytokine with a variety of functions across a wide range of biological processes. As such, it has been putatively implicated in the pathogenesis of innumerous diverse disease states, including infections, autoimmune disorders, cancers, neurodegenerative diseases and even drug dependencies, among many others. Involvement of TNF in the susceptibility to, development of, and outcomes associated with cardiovascular disease result largely from a reported role in vascular wound repair, insulin signalling and fat metabolism.

Results of previous studies suggest that common SNPs around the TNF locus exhibit allele-specific differences in gene transcription and biological measures. Skoog et $\mathrm{al}^{7}$ demonstrated that the minor (A) allele at the -863C/A SNP (rs1800630) was associated with a $31 \%$ reduction in reporter gene transcriptional activity in human hepatoblastoma cell lines compared with the major (C) allele. Allelespecific binding of monocytic and hepatic nuclear factors was also reported. These data were used to support their finding of reduced serum TNF-alpha levels in carriers of the A-allele. ${ }^{7}$ An Indian study likewise observed reduced serum TNF-alpha levels associated with the A-allele at the rs1800630 SNP. $^{8}$ In addition, these workers reported reduced circulating total serum IgE levels in carriers of the A-allele and used electrophoretic mobility shift assays to demonstrate allele-specific binding of endothelial cell derived nuclear extracts to the TNF promoter sequence. ${ }^{8} \mathrm{~A}$ recent study using nuclear extracts from human macrophage and THP-1 cell lines provided a molecular mechanism to account for these allele-specific differences in transcriptional activity; ${ }^{9}$ Skoog et al observed that the C-allele bound both the p50/p50 and p50/p65 dimer complexes of transcription factor nuclear factor-kappa $\mathrm{B}$ $(\mathrm{NF} \kappa \mathrm{B})$, whereas the A-allele only bound the p50/65 complex. ${ }^{9}$ Other sequence variations in the TNF promoter also appear to be functional. For example, the transcription factor OCT1 seems to bind the TNF-857T allele but not the $-857 \mathrm{C}$ (rs1799724), interacting both in vitro and 
in vivo with the $\mathrm{NF} \kappa \mathrm{B}$ p65 subunit at the adjacent binding site that includes the -863 nucleotide position. ${ }^{10}$ Despite this growing experimental evidence, the complete details as to how genetic sequence variation in and around TNF influences TNF-alpha expression, activity and downstream function remains to be fully elucidated, and inconsistencies regarding the impact of these polymorphisms (and TNF haplotypes) on blood TNF-alpha levels continues. ${ }^{11}$

Genetic variation around SERPINE is also thought to have functional implications. PAI-1 is a prothrombotic agent with structural similarities to other members of the serine proteinase inhibitor (Serpin) superfamily that includes angiotensinogen, alpha-1-antitrypsin and antithrombin III. PAI-1 has a role in response to vascular damage as it inhibits the plasmin activators t-PA and $\mathrm{u}-\mathrm{PA}$, which modulate the formation of plasmin from its precursor, plasminogen. It is an established marker of cardiovascular disease, with raised levels observed in MI patients. ${ }^{12}$ An insertion/deletion polymorphism in the SERPINE promoter (known commonly as the $4 \mathrm{G} / 5 \mathrm{G}$ variation, position $-675 \mathrm{bp}$ ) results in differences in the binding of $\mathrm{NF} \kappa \mathrm{B}$-like proteins to a glucocorticoid responsive element. ${ }^{13}$ The deletion allele (4G) leads to enhanced in vitro PAI-1 transcriptional activity and elevated plasma levels of PAI- $1 .{ }^{13}$ However, the latter finding is not universally observed and this inconsistency requires further resolution. $^{12}$

The literature also alludes to important potential interactions between the TNF and PAI-1 molecules. Exposure of 3T3-L1 adipocytes to TNF-alpha results in increased PAI-1 mRNA expression and PAI-1 protein levels; this induction is mediated (at least in response to chronic TNF-alpha exposure) via the $\mathrm{NF} \kappa \mathrm{B}$ pathway. ${ }^{14} \mathrm{~A}$ similar induction of fibrinolytic capacity by TNF-alpha in endothelial cells appears mediated by $\mathrm{NF} \kappa \mathrm{B}$ 's influence on regulatory sites within the t-PA gene. ${ }^{15}$ It is yet to be determined how genetic sequence variation in and around TNF or SERPINE impact on these molecular mechanisms.

The study of Hong et $a l^{6}$ curiously observed no significant influence of TNF gene variability on plasma TNF-alpha levels. However, this is not inconsistent with the prevailing literature, which reports such an association sporadically and with modest effects. ${ }^{11}$ These discrepancies in the literature may reflect the fact that TNF levels respond to a variety of environmental and lifestyle stimuli in a temporal manner, which is not consistently reproduced in the individual studies. It may also result from studyspecific differences in the sampling of subjects included in the various reports. For example, healthy subjects or those who have survived a single MI for more than a month are likely to experience different TNF-influencing stimuli to individuals with acute health problems or chronic illnesses. Thus, certain ascertainment methods may exclude individuals with the type of stimulation necessary to uncover or maximize any TNF gene effects.

In their hands, Hong et $a l^{6}$ observed that TNF haplotype and SERPINE genotype influenced PAI-1 plasma levels in vivo. Focused investigation revealed that these effects were restricted to individuals with a history of MI. Speculatively, this could simply mirror the fact that MI subjects are more likely to have experienced the type of acute or chronic TNF-alpha stimulation required to reveal the influence of TNF gene effects on PAI-1 levels. Such a state may be impacted by the underlying pathology of cardiovascular disease, factors associated with their increased risk for cardiovascular disease (for example smoking status, hypertension or lipid profile) or interventions instituted following diagnosis of illness (medical treatments, dietary or other lifestyle alterations such as exercise). Further study is required to explore these issues in more detail. It is interesting that the genotypic effects appear to result from a combination of unlike haplotypes. ${ }^{6}$ This suggests that there may be multiple sequence variables with functional implications, which have emerged over time on different ancestral backgrounds. It is also important to note that these genetic influences on PAI-1 could alternatively result from variations in other inflammation-related genes proximal to TNF, a chromosomal region equivocally reported to contain SNPs implicated in susceptibility in cardiovascular disease. ${ }^{16,17}$
The authors of the Hong study ${ }^{6}$ are correct to interpret their findings with caution awaiting confirmation of the results by others. The result could, of course, be due to chance. Although statistically robust for their study sample, the result could be deleteriously influenced by many uncontrollable biases inherent in even the most carefully designed studies, particularly given the multiple non-genetic influences on the biological traits being modelled. Fortunately, given the ready availability of requisite raw data already collected by many independent research groups, we will not have to wait long for other teams to confirm or contradict these interesting observations.

What are the implications of such study on the area of complex disease genetics? One of the epidemiological challenges in risk-factor determination is distinguishing between association and causality. Many biological, lifestyle and social markers of a disease stem either directly or indirectly from of the pathological process and are independent of its cause. One potential way to address this challenge is to identify the underlying genetic determinants of these markers within a population and test these factors in relation to a complex end point. An individual's genotype is assigned at conception, is with us for life and is, in most cases, largely independent of behavioural or environmental factors. Therefore, being able to establish whether such trait-influencing genetic variables are differentially represented in a group of patients with a disease enables confident inference that the trait itself contributes to risk for the disease. This is the basis of the concept of 'Mendelian randomisation' or 'Mendelian deconfounding' recently reviewed by Davey Smith et al. ${ }^{18,19}$ Furthermore, the knowledge of genetic influences in biological traits can provide important insights into how perturbations of these traits (via biological, pharmacological or sociological interventions) might likewise modify risk for disease. It is stating the obvious but if common genetic variables around TNF reduce the expression of TNF-alpha or influence downstream molecules like PAI-1, and these variables occur less frequently in individuals who develop cardiovascular disease, then interventions reproducing such effects could have positive effects on 
a population basis. These ideas are oversimplistic, however, as these genetic influences are likely to be pleiotropic (having multiple and possibly opposing influences on more than one pathway) that may affect cardiovascular diseaserelated outcomes or the risk for other complex phenotypes.

Unfortunately, we are yet to establish consensus with respect to the impact of TNF polymorphism on TNF-alpha or PAI-1. The extent to which elevated TNF or PAI-1 levels represent true risk factors for, as opposed to markers of, cardiovascular disease also remains unclear. Further focused work such as that presented by Hong et $a l^{6}$ will hopefully help to get to the heart of these matters

George D Mellick is at the Eskitis Institute for Cell and Molecular Therapies, Griffith University, Nathan Campus, Queensland 4111, Australia.

Tel: + 6173735 5019;

Fax: + 6173735 4070;

E-mail: G.Mellick@griffith.edu.au

\section{References}

1 Weiss KM, Terwilliger JD: How many diseases does it take to map a gene with SNPs? Nat Genet 2000; 26: 151-157.

2 Terwilliger JD, Hiekkalinna T: An utter refutation of the 'Fundamental Theorem of the HapMap'. Eur J Hum Genet 2006; 14: 426-437.

3 Buchanan AV, Weiss KM, Fullerton SM: Dissecting complex disease: the quest for the Philosopher's Stone? Int I Epidemiol 2006; 35: 562-571.

4 Stranger BE, Forrest MS, Clark AG et al: Genome-wide associations of gene expression variation in humans. PLoS Genet 2005; 1: e78.

5 Spielman RS, Bastone LA, Burdick JT, Morley M, Ewens WJ, Cheung VG: Common genetic variants account for differences in gene expression among ethnic groups. Nat Genet 2007; 39: 226-231.

6 Hong MG, Bennet AM, de Faire U, Prince JA: Phenotype selection for detecting variable genes: A survey of cardiovascular quantitative traits and TNF locus polymorphism. Eur J Hum Genet 2007; 15: 685-693.

7 Skoog T, van't Hooft FM, Kallin B et al: A common functional polymorphism $(\mathrm{C}->\mathrm{A}$ substitution at position -863) in the promoter region of the tumour necrosis factoralpha (TNF-alpha) gene associated with reduced circulating levels of TNF-alpha. Hum Mol Genet 1999; 8: 1443-1449.

8 Sharma S, Sharma A, Kumar S, Sharma SK, Ghosh B: Association of TNF haplotypes with asthma, serum IgE levels, and correlation with serum TNF-alpha levels. Am J Respir Cell Mol Biol 2006; 35: 488-495.

9 Skoog T, Hamsten A, Eriksson P: Allelespecific chromatin remodeling of the tumor necrosis factor-alpha promoter. Biochem Biophys Res Commun 2006; 351: $777-783$.

10 van Heel DA, Udalova IA, De Silva AP et al: Inflammatory bowel disease is associated with a TNF polymorphism that affects an interaction between the OCT1 and NF(-kappa)B transcription factors. Hum Mol Genet 2002; 11: 1281-1289.

11 Bennet AM, van Maarle MC, Hallqvist J et al: Association of TNF-alpha serum levels and TNFA promoter polymorphisms with risk of myocardial infarction. Atherosclerosis 2006; 187: 408-414.
12 Leander K, Wiman B, Hallqvist J, StenLinder M, de Faire U: PAI-1 level and the PAI-1 4G/5G polymorphism in relation to risk of non-fatal myocardial infarction: results from the Stockholm Heart Epidemiology Program (SHEEP). Thromb Haemost 2003; 89: 1064-1071.

13 Dawson SJ, Wiman B, Hamsten A, Green F, Humphries S, Henney AM: The two allele sequences of a common polymorphism in the promoter of the plasminogen activator inhibitor-1 (PAI-1) gene respond differently to interleukin-1 in HepG2 cells. J Biol Chem 1993; 268: 10739-10745.

14 Pandey M, Loskutoff DJ, Samad F: Molecular mechanisms of tumor necrosis factor-alpha-mediated plasminogen activator inhibitor-1 expression in adipocytes. FASEB J 2005; 19: 1317-1319.

15 Ulfhammer E, Larsson P, Karlsson L et al: TNF-alpha mediated suppression of tissue type plasminogen activator expression in vascular endothelial cells is NF-kappaBand p38 MAPK-dependent. J Thromb Haemost 2006; 4: 1781-1789.

16 Ozaki K, Ohnishi Y, Iida A et al: Functional SNPs in the lymphotoxin-alpha gene that are associated with susceptibility to myocardial infarction. Nat Genet 2002; 32: 650-654.

17 Yamada A, Ichihara S, Murase Y et al: Lack of association of polymorphisms of the lymphotoxin alpha gene with myocardial infarction in Japanese. J Mol Med 2004; 82: $477-483$.

18 Smith GD, Ebrahim S: Mendelian randomization: prospects, potentials, and limitations. Int J Epidemiol 2004; 33: $30-42$.

19 Davey Smith G, Ebrahim S, Lewis S, Hansell AL, Palmer LJ, Burton PR: Genetic epidemiology and public health: hope, hype, and future prospects. Lancet 2005; 366: $1484-1498$. 